\title{
New Perspectives in the Diagnosis and Management of Allergic Fungal Airway Disease
}

\author{
Andrew J Wardlaw (D) \\ Eva-Maria Rick' \\ Leyla Pur Ozyigit ${ }^{2}$ \\ Alys Scadding ${ }^{2}$ \\ Erol A Gaillard (1D ${ }^{3}$ \\ Catherine H Pashley (D)
}

'Institute for Lung Health, Department of Respiratory Sciences, College of Life Sciences, University of Leicester, and Allergy and Respiratory Medicine Service, NIHR Biomedical Research Centre: Respiratory, University Hospitals of Leicester NHS Trust, Leicester, UK; ${ }^{2}$ Allergy and Respiratory Services University Hospitals of Leicester NHS Trust, Leicester, UK; ${ }^{3}$ Institute for Lung Health, Department of Respiratory Sciences, College of Life Sciences, Department of Paediatrics, NIHR Biomedical Research Centre: Respiratory, University Hospitals of Leicester NHS Trust, Leicester, UK

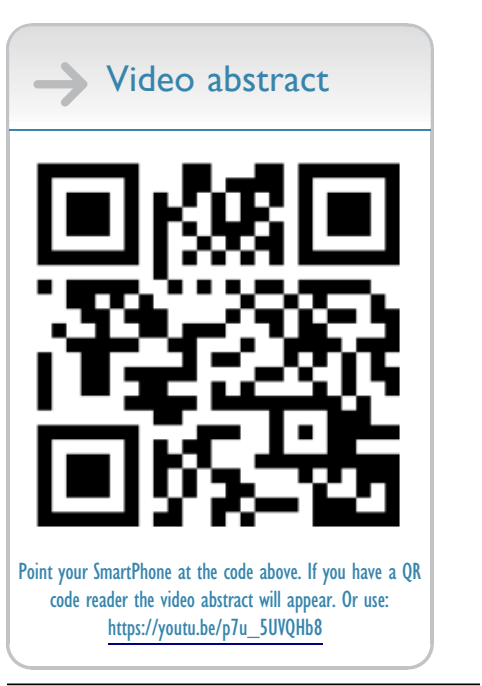

Correspondence: Andrew J Wardlaw Glenfield Hospital, Groby Road, Leicester, LE3 9QP, UK

Tel +44 I I6 258384 I

Email aw24@le.ac.uk

\begin{abstract}
Allergy to airway-colonising, thermotolerant, filamentous fungi represents a distinct eosinophilic endotype of often severe lung disease. This endotype, which particularly affects adult asthma, but also complicates other airway diseases and sometimes occurs de novo, has a heterogeneous presentation ranging from severe eosinophilic asthma to lobar collapse. Its hallmark is lung damage, characterised by fixed airflow obstruction (FAO), bronchiectasis and lung fibrosis. It has a number of monikers including severe asthma with fungal sensitisation (SAFS) and allergic bronchopulmonary aspergillosis/mycosis (ABPA/ $\mathrm{M})$, but these exclusive terms constitute only sub-sets of the condition. In order to capture the full extent of the syndrome we prefer the inclusive term allergic fungal airway disease (AFAD), the criteria for which are IgE sensitisation to relevant fungi in association with airway disease. The primary fungus involved is Aspergillus fumigatus, but a number of other thermotolerant species from several genera have been implicated. The unifying mechanism involves germination of inhaled fungal spores in the lung in the context of $\operatorname{IgE}$ sensitisation, leading to a persistent and vigorous eosinophilic inflammatory response in association with release of fungal proteases. Most allergenic fungi, including Alternaria and Cladosporium species, are not thermotolerant and cannot germinate in the airways so only act as aeroallergens and do not cause AFAD. Studies of the airway mycobiome have shown that A. fumigatus colonises the normal as much as the asthmatic airway, suggesting it is the tendency to become IgE-sensitised that is the critical triggering factor for AFAD rather than colonisation per se. Treatment is aimed at preventing exacerbations with glucocorticoids and increasingly by the use of anti-T2 biological therapies. Anti-fungal therapy has a limited place in management, but is an effective treatment for fungal bronchitis which complicates AFAD in about $10 \%$ of cases.
\end{abstract}

Keywords: fungi, Aspergillus, ABPA, SAFS, asthma, eosinophils

\section{Introduction}

Fungal lung disease represents a heterogeneous group of conditions (Figure 1). Broadly speaking they can be divided into infective, toxic or allergic in nature, although there is a degree of overlap. The allergic group can again be broadly divided into two, both of which can be associated with severe asthma. The first type is an allergenic response to environmental fungi such as Alternaria and Cladosporium which act as seasonal aeroallergens, akin to grass pollen exposure, the symptoms of which are directly related to airborne concentrations of fungal material and which can include acute severe exacerbations. The second type involves an allergic response to thermotolerant filamentous fungi such as species from the Aspergillus and Penicillium genera which, while they can also act as 


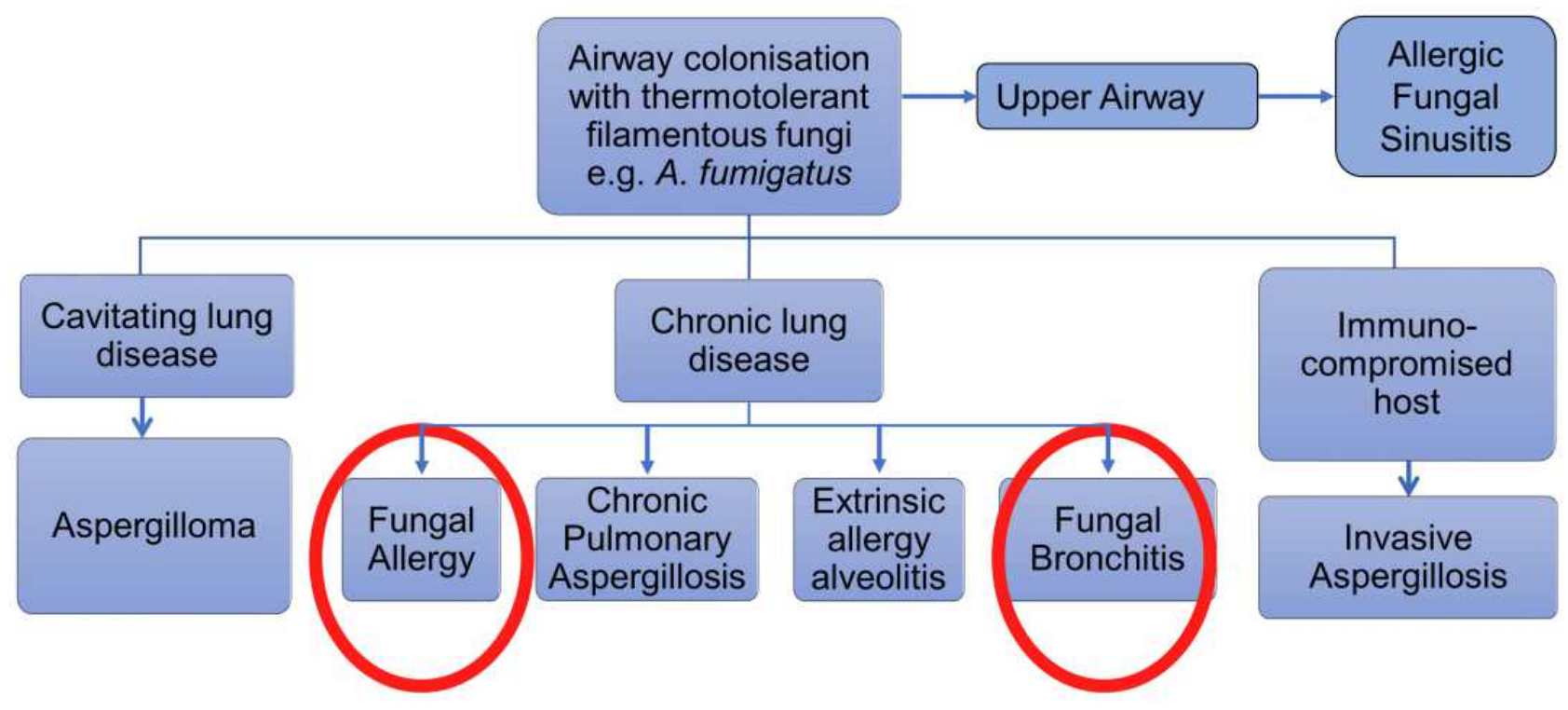

Figure I Various presentations of fungal involvement in lung disease.

aeroallergens, have the additional property of being able to germinate in the airways, colonising the lungs and causing a persistent allergenic stimulus that can lead to lung damage. ${ }^{1-3}$ In addition, allergic reactions, or at least generation of specific $\operatorname{IgE}$, to mucosal colonising yeasts and skin fungi such as Candida, Malassezia and Trichophyton species are common, particularly in those with atopic disease, but of less certain clinical relevance to the lung. This review is largely concerned with the clinical effects of allergy to thermotolerant, filamentous respiratory fungi.

The variability in the presentation of fungal allergy to thermotolerant fungi has caused confusion in terms of diagnostic labelling which has obstructed a clear understanding of the role played by these fungi in lung disease. ${ }^{4}$ Most authorities have taken an exclusive approach, parcelling out distinct presentations such as allergic bronchopulmonary aspergillosis/mycosis (ABPA/M) and severe asthma with fungal sensitisation (SAFS), as if they were separate conditions. As there is limited evidence that there are distinct mechanisms involved in the spectrum of thermotolerant fungal lung allergy, we prefer an inclusive set of criteria which includes all presentations of the disease under the umbrella term allergic fungal airway disease (AFAD), the rationale for which we have discussed in the first section. The relationship between these various terms is illustrated in Figure 2. We then discuss the aerobiology of relevant fungi including component-resolved approaches to fungal allergens, followed by sections on pathogenic pathways related to disease causation, and new insights into the fungal lung mycobiome. Subsequently we describe the clinical presentation and management of AFAD in adults and children including the role of antifungal therapy.

Evidence that fungal allergy is driving a particular clinical condition such as AFAD (or for that matter ABPA and SAFS), is circumstantial, based on close association and plausibility. More definitive proof of a causal link would require a specific intervention such as immunotherapy, and we are not aware of any controlled clinical trials that have used this approach for AFAD. The other difficulty in proving an association is the length of time that it takes for lung damage secondary to fungal allergy to develop. The only situation relevant to this review where a specific intervention has clearly (at least to these investigators' satisfaction) shown a direct relationship between the fungal involvement and disease is in the case of fungal bronchitis causing exacerbations of airway disease where specific anti-fungal pharmacotherapy can be very effective. ${ }^{5}$

\section{Semantics and Definitions}

Names for disease processes are ideally based on a welldefined pathogenetic pathway that explains the clinical features, prognosis and response to treatment. Examples in lung disease include cystic fibrosis and tuberculosis. In such conditions the presence of the disease can be confirmed with a high degree of specificity and sensitivity, although it is notable that there is still considerable heterogeneity in clinical presentation. When there is insufficient knowledge of 


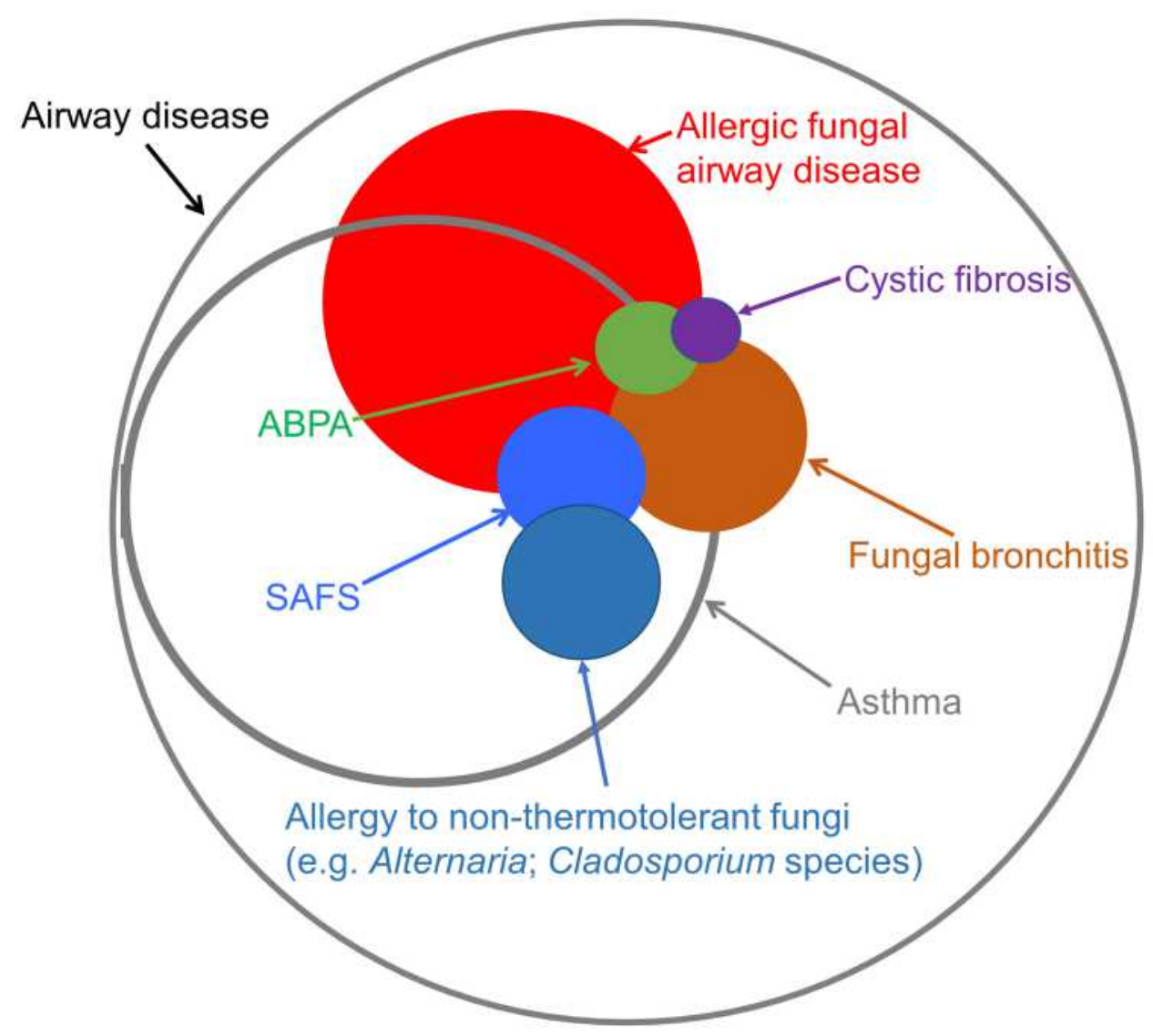

Figure 2 A Venn diagram showing the relationship between the various terms used to describe AFAD.

aetiology the question of what constitutes the disease often arises. Historically, anecdotal labels, often based on single observable characteristics (phenotypes) (eg exercise-induced asthma), have been used to describe heterogeneity. More recently, multi-dimensional and unbiased approaches to interrogating disease heterogeneity using complex biostatistical analyses such as cluster analysis and machine learning have been employed to reveal patterns of disease more closely related to disease mechanisms (endotypes) and deconstruct airway disease into its component pathophysiological components. $^{6-9}$

The field of fungal allergy in lung disease has followed the tradition of descriptive labelling, based largely on anecdotal phenotypic observations in biased populations. Thus ABPA/M, defined by a restrictive set of criteria with relatively arbitrary cut-offs, identified a small subset of patients according to a florid $\mathrm{T} 2$ immune response to certain fungi, particularly A. fumigatus. ${ }^{10,11}$ This term has become indelibly embedded in the literature, although the lack of a clear statistical basis for the criteria has led to uncertainty amongst both investigators and clinicians about what exactly is meant by ABPA/M, with the criteria often honoured in the breach. ${ }^{12}$ Recent attempts to refine the criteria to make them more representative of clinical experience have been hampered by the lack of a gold standard. This has resulted in tautology where subjects with ABPA/M are preselected using characteristics which are then incorporated into the diagnostic criteria. ${ }^{13,14}$ There is general agreement that the condition which comes under various headings (ABPA/M, SAFS, AFAD, airway mycosis or fungal asthma) is due to an immunological response to lung-colonising, thermotolerant, filamentous fungi, marked by IgE sensitisation to genera such as Aspergillus and Penicillium, with mucus plugging likely to be an important factor in pathogenesis. ${ }^{15}$ This process usually, though not invariably, occurs in the context of another airway disease, particularly asthma or cystic fibrosis, but also COPD and primary bronchiectasis. It leads over a prolonged period, punctuated by exacerbations, to lung damage (fixed airflow obstruction (FAO), bronchiectasis and lung fibrosis). ${ }^{16-19}$ Presentation is variable both in the pattern of symptoms and signs but also severity, and all patients with specific IgE to Aspergillus 
fumigatus are at risk of developing the condition. ${ }^{20} \mathrm{ABPA} /$ $\mathrm{M}$ captures only $\sim 10 \%$ of people at risk. In an attempt to address this issue in the context of asthma, Denning et al introduced the term SAFS which was defined in opposition to ABPA/M. ${ }^{1,21}$ However, the exclusion of subjects with severe asthma who meet the criteria for ABPA has led to clinical confusion with the use of the oxymoron ABPASAFS. ${ }^{22}$ Moreover "severe asthma" is a vexed term which defies a clear definition, and focusing on asthma excludes people with other airway diseases or none. ${ }^{23}$ Perhaps most significantly the inclusion of people with IgE sensitisation to yeasts and non-lung colonising fungi which are unlikely to be involved in the pathogenesis of the condition is problematic.

For these reasons, until we have better biomarkers for the risk of developing lung damage from fungal allergy, we prefer an inclusive term such as AFAD, the criteria for which are simply IgE sensitisation to relevant fungi and airway disease. ${ }^{24}$ The main criticism of this approach is that it is too inclusive, including people with mild, clinically unimportant disease, although this does not take into account the slow progression of the condition over decades. Better biomarkers of the risk of developing troublesome disease are certainly required, but until then the qualification of AFAD with the degree of severity (mild, moderate, severe) should suffice (Table 1).

Allergic fungal rhinosinusitis (AFRS) is a parallel condition to lower airway disease with many features in common with AFAD, including the uncertainty of when IgE sensitisation and fungal detection are clinically relevant. ${ }^{25}$ AFRS is currently regarded as a complication of chronic sinusitis (CRS) and is characterised by viscid eosinophilic mucus, characteristic appearances on the CT scan and non-invasive fungal colonisation. Criteria for diagnosis were established by Bent and Kuhn in 1994, although this was on the basis of only 15 patients. ${ }^{26}$ The epidemiology is uncertain, although it appears less common overall than lower airway fungal disease and is more often diagnosed in a younger age group. As with AFAD the incidence may vary with geography and climate. Surgical clearance supported by corticosteroids is the mainstay of management.

\section{Aerobiology of Thermotolerant Filamentous Fungi}

Fungi are one of the kingdoms of life, with approximately 120,000 species currently identified and suggestions that the true number may be well over a million. ${ }^{27}$ They are eukaryotic, non-chlorophyllous organisms, dependent on external sources for nutrition. They exist as saprophytes, symbionts or parasites on animals and plants, ubiquitously in all environmental niches including the human body. Many sporulate, and every inhalation can contain large amounts of fungal spores and hyphae from diverse sources. ${ }^{28}$ Airborne fungal spore counts are 100-1000 times greater than those of pollen. The phenotype of fungi ranges from unicellular to filamentous (moulds). Most filamentous fungi belong to two families, Ascomycota and Basidiomycota. They have sexual and asexual forms which can be pleomorphic and carry quite different names, which confuses their description. Human pathogenicity is restricted to a few hundred species, and while eighty genera have been reported as potentially causing allergic disease, allergenic proteins have only been identified in about a third of these. ${ }^{29}$ A comprehensive appreciation of the extent of species causing fungal allergy is hampered by the paucity of suitable reagents for testing. ${ }^{30} \mathrm{~A}$ number of fungi are commensals in that they can routinely be cultured from mucosal surfaces where they do no harm. Candida species are found routinely in the oropharynx, gut and vaginal tracts, whereas Malassezia and Trichophyton species occur on the skin. In some circumstances these commensal fungi can cause disease, through both allergenic and infective mechanisms. ${ }^{30-33}$ The optimal growth temperature for most fungi is $25-30^{\circ} \mathrm{C}$. Apart from the skin, potential for infection requires a greater degree of thermotolerance to allow them to germinate at body temperature. This is a particular property of fungi involved in decomposition of plant material, such as members of the Aspergillus, Penicillium and Paecilomyces genera, which produce a range of the necessary proteases for digestion of plant material. ${ }^{34}$ Large numbers of spores are generated in this setting, which can be a particular problem for gardeners and industrial composting facilities where strict regulations are imposed to control exposure. ${ }^{35}$ While plant pathogens, typically Alternaria and Cladosporium species, but also including a wide variety of spore-bearing fungi, are present in much higher quantities in outdoor air in the summer and autumn, the concentrations of Asp/Pen spores in outdoor air are fairly constant at low levels throughout the year. Indoor exposure to spores can result from leakage from the outside air or from indoor sources. ${ }^{36} \mathrm{~A}$ wide variety of fungi are found growing in damp indoor 
Table I Indicators of Markers of Severity of AFAD (a Positive for Any of the Parameters Would Be Sufficient to Record AFAD as Severe)

\begin{tabular}{|c|c|c|c|}
\hline & Mild & Moderate & Severe \\
\hline Clinical & $\begin{array}{l}\text { Intermittent non-productive cough } \\
\text { and wheeze } \\
\text { No exacerbations } \\
\text { No antibiotics } \\
\text { GINA I }\end{array}$ & $\begin{array}{l}\text { Occasional cough with sputum } \\
\text { Frequent wheeze/SOB } \\
\text { Occasional exacerbations } \\
\text { Occasional need for antibiotics } \\
\text { GINA } 2 / 3\end{array}$ & $\begin{array}{l}\text { Frequent productive cough } \\
\text { Daily wheeze/SOB } \\
\text { Frequent exacerbations and need for } \\
\text { antibiotics } \\
\text { Fungal bronchitis } \\
\text { GINA } 4 / 5\end{array}$ \\
\hline Physiology & Post-bronchodilator FEV $>$ > $90 \%$ & Post-bronchodilator FEV, $70 \%-90 \%$. & Post-bronchodilator FEV $<<70 \%$ \\
\hline Radiology & Normal CXR and chest CT scan & Occasional fleeting shadows & $\begin{array}{l}\text { Bronchiectasis } \\
\text { Lung fibrosis } \\
\text { Tree-in-bud shadowing } \\
\text { Hyper-attenuated mucus on CT scan } \\
\text { Eosinophilic pneumonia } \\
\text { Lobar collapse }\end{array}$ \\
\hline Prognosis & $\begin{array}{l}\text { Presumed very good but advisable to } \\
\text { monitor lung function long term }\end{array}$ & $\begin{array}{l}\text { Possibility of lung damage and associated } \\
\text { symptoms becoming more marked over time }\end{array}$ & $\begin{array}{l}\text { Associated with considerable morbidity } \\
\text { and increased premature mortality }\end{array}$ \\
\hline
\end{tabular}

environments including several Aspergillus and Penicillium species. $^{37}$

Asp/Pen spores are less than $5 \mu \mathrm{m}$ in diameter and can therefore penetrate to the deeper parts of the lung. However, they are relatively inert, and there is evidence that it is only when the spores germinate that they become pathogenic. $^{38}$ Relating exposure to bioaerosols to health effects is difficult, and the important question of the relationship between exposure and disease in AFAD is uncertain, although there seems a more clear-cut association with hypersensitivity pneumonitis. ${ }^{39-41}$ People already sensitised to A. fumigatus are without doubt at risk of significant harm if they are exposed to high concentrations of fungal material, for example during certain gardening activities. However, the extent to which high levels of exposure are a risk factor for IgE sensitisation is less clear. Studies on the health effects of flooding events in the USA have been conflicting, and while there is a general trend towards a link between atopic disease and fungal exposure, at least in children, this is not clearcut and is not often related to risk of IgE sensitisation. ${ }^{42-44}$

\section{Fungal Allergens}

Although Gell and Coombs (G\&C) type III and IV hypersensitivity have been implicated in fungal allergy, and are certainly involved in causing fungal mediated hypersensitivity pneumonitis, the major burden of disease on current evidence is linked to $\mathrm{G \& C}$ type 1 hypersensitivity characterised by IgE sensitisation and T2-mediated eosinophilic inflammation. As noted above, a specific IgE to A. fumigatus is the most inclusive biomarker of AFAD, with the caveat that this does not automatically mean that Aspergillus is the cause of the patient's clinical phenotype. Yeasts such as Candida spp., which are both thermotolerant and a common cause of IgE sensitisation, do not seem to cause the same pattern of disease as the filamentous fungi, the reasons for which are unclear. ${ }^{29}$ As with other sources of allergens an individual fungal species will contain many potential allergenic proteins grouped into families. ${ }^{45}$ In other allergic diseases, in particular food allergy, the use of recombinant allergens to determine the individual proteins to which a person is sensitised has improved the specificity of allergens as biomarkers of disease severity, an approach termed component-resolved diagnosis. ${ }^{46}$ However, a similar approach to fungal allergy is in its infancy, in large part because of lack of reagents, with only the allergens of A. fumigatus, Asp f1-4 and Asp f6 commercially available for testing. This is important, as the lack of significant disease in some people sensitised to A. fumigatus extracts could be in part because the sensitisation is secondary to a cross-reacting allergen, for example from Malassezia species in someone with atopic dermatitis. ${ }^{47,48}$ We found that of the five recombinant allergens of $A$. fumigatus mentioned above, Asp f3 and f4 were the most closely associated with bronchiectasis in patients with AFAD (none of the allergens were associated with fixed airflow obstruction (FAO)). ${ }^{49}$ 
Identification and detailed characterisation of fungal allergens are complex, difficult and time-consuming. While 23 allergens of $A$. fumigatus are listed on the WHO website and 81 were identified using phage display, relatively few have been fully characterised. ${ }^{30,50}$ Patients who fit the criteria for ABPA have been shown to have higher titres of IgE antibodies against the commercially available A. fumigatus recombinant allergens than "non-ABPA asthma", but this is not surprising as ABPA represents a sub-set of AFAD with a florid T2 immunological response to $A$. fumigatus and consequent high levels of total and specific IgE. ${ }^{51,52}$ What is generally lacking are studies relating titres of the antibodies against recombinant allergens to clinically relevant outcomes. Recombinant fungal allergens which track more closely to lung damage than crude extracts would aid assessment of prognosis, provide clues regarding pathogenesis and offer the opportunity of becoming effective agents for immunotherapy to prevent the development of fixed airflow obstruction and bronchiectasis.

\section{Pathogenesis of AFAD}

While $A$. fumigatus and related colonising fungi can cause a range of lung ailments as well as being present without causing disease, the hallmark of AFAD is exaggerated T2 immunity causing $\operatorname{IgE}$ sensitisation to filamentous fungi and eosinophilic inflammation (Figure 3). The mechanisms involved in inflammatory responses in the context of T2-mediated airway disease, particularly asthma, have been exhaustively researched. ${ }^{53,54}$ The question is what, if anything, is specific about fungal immune responses in AFAD. Most research into fungal disease in the lung has focused on the role of innate and adaptive immune mechanisms in preventing invasive infection. ${ }^{55}$ Bacher et al found that intestinal C. albicans was a major inducer of Th17 cells in humans, that these antigen specific cells were cross-reactive with $A$. fumigatus and were induced in the lung during exacerbations of ABPA. ${ }^{56}$ Whether Th17 cells are involved in the pathogenesis of AFAD is not clear, although they have been identified in asthmatic

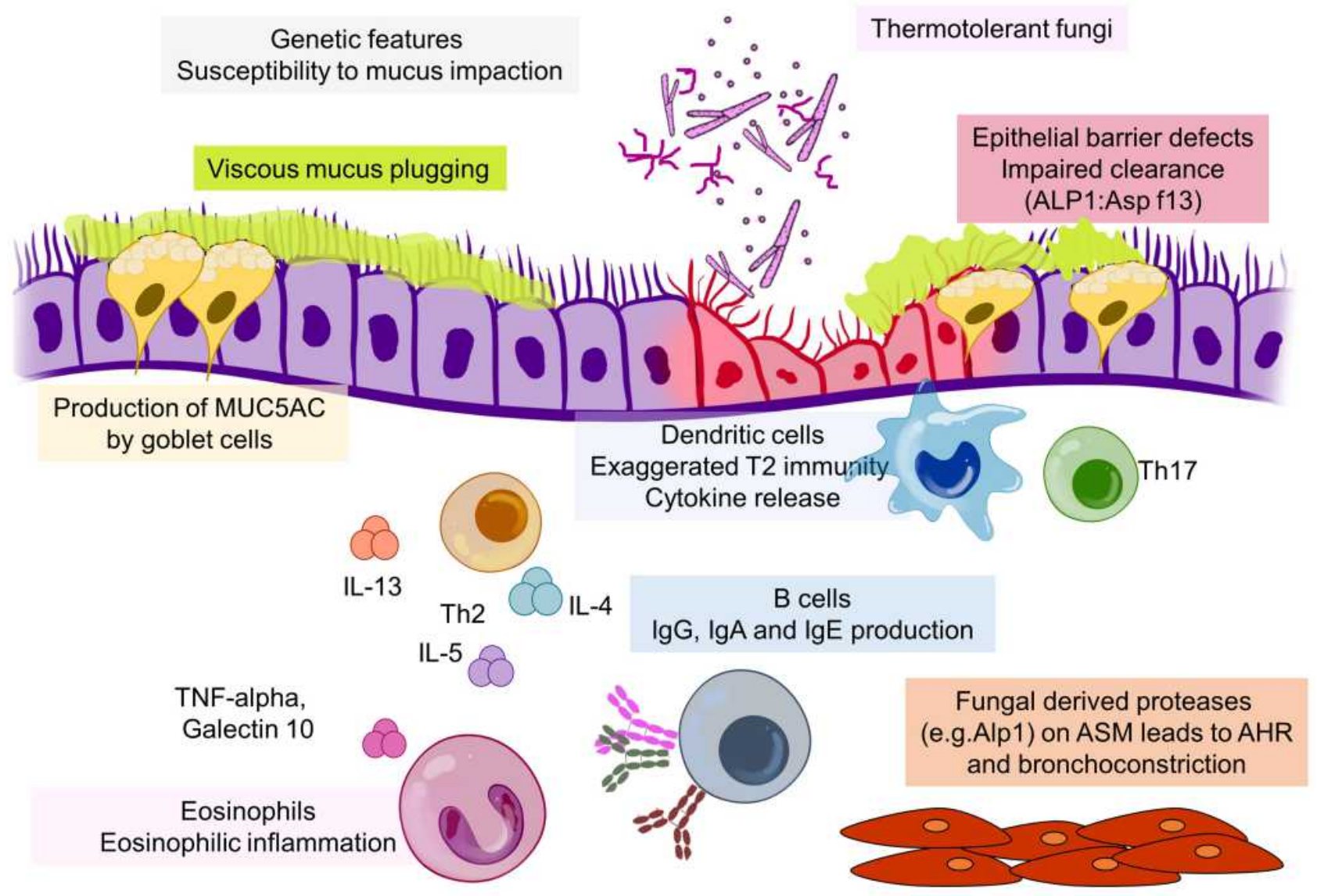

Figure 3 Schematic diagram outlining the pathways involved in the pathogenesis of AFAD. 
airways where they were mutually exclusive with Th2 cells. ${ }^{57,58}$ The most obvious pathology in AFAD is in the lumen of the airway with production of viscid mucus a characteristic feature, and there is limited evidence of fungal invasion within the bronchial mucosa. ${ }^{59}$ However, fungi including A. fumigatus contain a range of proteases, which is a common factor in protein allergenicity. ${ }^{60} \mathrm{An}$ interesting literature is developing around a potential role for Alp1 (the allergen Asp f13), an alkaline serine protease and the most abundant protein secreted by A. fumigatus. ${ }^{61,62}$ In a mouse model Alp1 caused a T celldependent lung eosinophilia as a result of damage to the cell-cell junctions of club cells within the bronchiolar epithelium via a TRVP4 calcium ion channel-dependent mechanism. ${ }^{63}$ Balenga et al, also in a mouse model of asthma, found that Alp1 promoted airway hyperresponsiveness by disrupting airway smooth muscle-extracellular matrix interactions. ${ }^{64}$ The group later demonstrated that the amount of Alp1 in the lower airways of asthmatics correlated with severity of disease, and interestingly this was not associated with IgE sensitisation to A. fumigatus. However, Alp1 concentrations were higher in sputum from sensitised asthmatics. ${ }^{65}$ Viscid mucus can be detected and measured by multidetector CT scans or MRI and is related to severity, although this is also a feature of severe eosinophilic asthma. ${ }^{66-71}$ Mucus impaction in AFAD is most strikingly evident in those patients who present with lobar collapse due to inspissated mucus, but is also seen in the smaller airways on CT scans and noticeable clinically as expectoration of rubbery sputum plugs. The pathogenesis of bronchiectasis is multi-factorial and complex, with a number of causes related to impaired local and systemic immunity, but defects in mucociliary clearance are often a common factor. ${ }^{72}$ It is well established that persistent airway blockage leads to bronchiectasis, and it is possible that the mucus impaction in AFAD is the major reason for the development of bronchiectasis. ${ }^{73,74}$ The fleeting shadows that are also characteristic of AFAD are also probably secondary to mucus obstruction leading to a localised area of eosinophilic pneumonia. The precise pathway by which $\operatorname{IgE}$ sensitisation to thermotolerant filamentous fungi may cause production of viscid mucus is not clear, but could be related to excess production of MUC5AC by goblet cells as a result of vigorous $\mathrm{T} 2$ hyperimmune stimulation. ${ }^{68,75}$ IL-13 is a known stimulator of mucus production, but eosinophils through the generation of TNF-alpha and galectin 10 (Charcot-Leyden protein) stimulating the generation of MUC5AC may be a more potent and direct cause. ${ }^{76,77}$ Eosinophil extracellular traps may also be a contributory factor leading to release of viscid genomic DNA into the lumen. ${ }^{78,79}$ In addition eosinophils may also increase the elasticity of mucus by causing oxidation of cysteine residues in MUC5AC via EPO. ${ }^{70}$ The concept of a direct role for eosinophils in AFAD is supported by the effectiveness of mepolizumab, an anti-IL -5 biological therapy in preventing exacerbations. ${ }^{80}$ The causes of fixed airflow obstruction and fibrosis are less clear-cut. Asp f3 and f4 which were associated with bronchiectasis were not associated with impairment in lung function. ${ }^{49}$ FAO in AFAD is presumably caused by pathology in the small airways, although the inflammatory profile appears distinct from smoking-related COPD, a condition which many patients with AFAD are labelled as having when FAO is prominent. ${ }^{81}$ Eosinophils are potent inducers of fibrotic reactions, and it is this pathway that might be responsible both for AFAD-associated fixed airflow obstruction and lung fibrosis, with IgE sensitisation acting as a marker of eosinophil production and activation rather than directly causing the pathology. ${ }^{82-84}$ The reasons why fungal proteins are a common cause of $\operatorname{IgE}$ sensitisation are of interest. ${ }^{85}$ The HLA genotype of an individual is also likely to be a factor. ${ }^{86}$ However, despite a considerable amount of research into the genetics of both airway disease and lung function there have been at best only weak signals related to AFAD. ${ }^{2,87}$ This would suggest that environmental factors are paramount in the development of AFAD.

There is considerable variability in the severity of AFAD, with some people sensitised to A. fumigatus and related fungi having very mild disease and others being severely affected with a major effect on morbidity and even mortality. This heterogeneity is a feature of all airway and allergic diseases and indeed chronic disease in general even where the aetiological factor is known. Such variability in severity is likely to be due to a complex combination of factors. These include environmental factors, such as the degree of fungal exposure, factors related to the species and strain of fungus and the degree of colonisaton and host factors, such as the susceptibility to fungal mycotoxins, the degree of immunological sensitivity and, as noted above in the allergens section, the site and source of sensitisation.

\section{The Airway Mycobiota}

There is general agreement that colonisation of the airways with germinating filamentous fungi is an important 
component of the pathophysiology of AFAD. Identifying and quantifying the fungi present in the lower airways should therefore be fundamental to understanding the relationship between colonisation and disease. However, this is not straightforward, and there are no universally accepted protocols to determine fungal load in the lungs.

\section{Fungal Culture}

Culture is the usual method for determining what potential pathogens are present in airway secretions. This provides confident identification, an estimate of the amount present and reassurance that the pathogen detected is viable, although this is less relevant for allergic reactions. The success of culture is highly dependent on the volume and quality of material plated on the culture medium. The methodology for detecting fungi, at least in the UK National Health Service, is relatively insensitive and fails to detect fungi in many cases. ${ }^{88,89}$ In addition there is usually limited effort put into detecting and characterising fungi other than Aspergillus genera, and the clinical relevance of a positive culture of Candida species is often downplayed. ${ }^{90}$ Plating sputum plugs directly on to culture media, we found a positive culture at $37^{\circ} \mathrm{C}$ for 27 different taxa of filamentous fungi in the sputum of $57 \%$ of moderate-to-severe asthmatics, with the great majority of isolates being A. fumigatus. Rates of positive culture were higher in subjects sensitised to A. fumigatus and P. chrysogenum, but not to the non-colonising Alternaria, Cladosporium or Botrytis. Culture rates in healthy subjects were low, with one out of eighteen subjects testing positive for A. fumigatus. Subjects with a positive sputum culture sensitised to A. fumigatus had a $20 \%$ reduction in postbronchodilator $\mathrm{FEV}_{1}$ compared to non-sensitised, culturenegative individuals. ${ }^{91}$ Similar rates of culture were seen in COPD, although without the association with reduced lung function. ${ }^{17}$ In a longitudinal study of 68 patients with non-cystic bronchiectasis recruited from a specialist clinic (four of whom had an existing label of ABPA), 23\% were IgE-sensitised to A. fumigatus. Of the 52 subjects able to produce sputum, 33 participants did not culture any fungus, and 19 (37\%) had a positive fungal culture. All eight of the participants who produced sputum and were IgEsensitised to A. fumigatus were fungal culture-positive, emphasising the association of sensitisation with culture noted above and by others in asthma. ${ }^{92}$ The participants with a positive sputum fungal culture had significantly more symptoms of cough, breathlessness, sputum production and sputum purulence compared to those with negative fungal cultures. ${ }^{93}$ Using potato dextrose agar containing chloramphenicol $(16 \mu \mathrm{g} / \mathrm{mL})$, gentamicin (4 $\mu \mathrm{g} / \mathrm{mL}$ ) and fluconazole $(5 \mu \mathrm{g} / \mathrm{mL})$ (PGCF) as a culture medium, the most commonly cultured fungi were the Aspergillus species, of which A. fumigatus was the most commonly cultured. Penicillium species were the next prevalent, with a range of other fungi identified as single isolates (Table 2).

Table 2 Fungal Species Cultured from Patients with Non-Cystic Bronchiectasis

\begin{tabular}{|c|c|c|}
\hline Culture Medium & $\begin{array}{l}\text { Fungal } \\
\text { Species }\end{array}$ & $\begin{array}{l}\text { Number } \\
\text { of Isolates }\end{array}$ \\
\hline \multirow{7}{*}{$\begin{array}{l}\text { Potato dextrose agar (containing } \\
\text { chloramphenicol }(16 \mu g / \mathrm{mL}) \text {, } \\
\text { gentamicin }(4 \mu \mathrm{g} / \mathrm{mL}) \text { and } \\
\text { fluconazole }(5 \mu \mathrm{g} / \mathrm{mL}))(P G C F)(I)\end{array}$} & $\begin{array}{l}\text { Aspergillus } \\
\text { fumigatus }\end{array}$ & 56 \\
\hline & Aspergillus niger & 7 \\
\hline & $\begin{array}{l}\text { Aspergillus } \\
\text { flavus }\end{array}$ & 4 \\
\hline & $\begin{array}{l}\text { Aspergillus } \\
\text { nidulans }\end{array}$ & 2 \\
\hline & Penicillium spp. & 5 \\
\hline & Other & 15 \\
\hline & No growth & 100 \\
\hline \multirow[t]{10}{*}{$\begin{array}{l}\text { Scedosporium selective agar (SceSel } \\
+)(2)\end{array}$} & $\begin{array}{l}\text { Gloeophyllum } \\
\text { trabeum }\end{array}$ & I \\
\hline & $\begin{array}{l}\text { Coprinellus } \\
\text { xanthothrix }\end{array}$ & I \\
\hline & $\begin{array}{l}\text { Hyphodontia } \\
\text { microspora }\end{array}$ & 2 \\
\hline & $\begin{array}{l}\text { Rhizomucor } \\
\text { miehei }\end{array}$ & 2 \\
\hline & $\begin{array}{l}\text { Coprinopsis } \\
\text { cinerea }\end{array}$ & 1 \\
\hline & $\begin{array}{l}\text { Phanerochaete } \\
\text { chrysosporium }\end{array}$ & 1 \\
\hline & $\begin{array}{l}\text { Talaromyces } \\
\text { spp. }\end{array}$ & 4 \\
\hline & $\begin{array}{l}\text { Phanerochaete } \\
\text { sordida }\end{array}$ & 1 \\
\hline & $\begin{array}{l}\text { Agaricaceae } \\
\text { spp. }\end{array}$ & I \\
\hline & Other & 3 \\
\hline
\end{tabular}


Quantification of fungal load can be achieved to an extent by counting the number of fungal colonies on a culture plate, but for technical reasons this is more useful for yeast species. Any positive culture of filamentous fungi could be potentially clinically significant.

\section{Molecular Techniques}

Molecular approaches including quantitative PCR could offer a better solution for quantifying fungal load in the airways, and a number of testing kits are commercially available, but are not yet in general use. ${ }^{94}$ Our experience with this approach to measuring fungal load has not been productive. Galactomannan is not a useful measurement to quantify fungi in sputum. ${ }^{95}$ Not all fungi are readily culturable, and fast-growing species may outcompete slowergrowing fungi. Next-generation sequencing using universal primers can obviate the drawbacks of culture. We used this approach on sputum and bronchoscopy samples from subjects with asthma and healthy controls, comparing outcomes with environmental samples to separate inhaled fungi from those colonising the airways. ${ }^{96}$ We detected a very diverse range of fungi, but most were at very low levels. The profile was dominated by A. fumigatus, Candida albicans and Cladosporium. The last-mentioned was probably present as a result of inhalation. Surprisingly there was no difference in A. fumigatus or C. albicans between asthmatics with and without IgE sensitisation to A. fumigatus and healthy controls in either the percentage of people where the fungi were found or the number of reads per positive individual which is a relative measure of the amount of each of the fungi present. This was similar to the finding by Sullivan et al who used PCR to detect A. fumigatus in BAL in asthma and did not find any relationship between a positive signal for the mould with either sensitisation or disease severity. ${ }^{97}$ In our microbiome study there was, however, variability between different airway compartments, with the epithelial compartment standing out as distinct. In particular we noted a strong signal from Aspergillus tubingensis whose presence was inversely associated with neutrophilic inflammation, suggesting a hitherto unexpected role for this species in asthma pathogenesis which is worthy of further investigation. ${ }^{98,99}$ The response of the bronchial epithelium to fungi could be critical, as suggested by Gago et al who demonstrated that a host variant in ZNF77 (a zinc finger transcription factor) increased adhesion of A. fumigatus to an epithelial cell line and that patients with ABPA who were heterozygous for the variant had a higher fungal load in bronchoalveolar lavage. ${ }^{100}$ Our study demonstrated that A. fumigatus colonises the healthy respiratory tract and conflicted with culture data which demonstrated low rates of positive culture in healthy subjects in both sputum and bronchoalveolar lavage (BAL). The molecular approach may be picking up DNA material that is non-viable and not indicative of active colonisation. Fraczek et al using a similar approach also found significant amounts of A. fumigatus and Candida spp. in the airways of healthy subjects, although there was a suggestion of a higher burden in asthma measured by qPCR. ${ }^{101}$ They also found a link between fungal load and corticosteroid dose, although this was confounded by disease severity. The extent to which corticosteroids might increase the risk of fungal colonisation in the lower airways as opposed to the oropharynx, particularly with Candida spp., is uncertain. ${ }^{97}$ We have not found a close association between dose of inhaled corticosteroids (ICS) and the rate or amount of positive culture in our studies, but it is very difficult to gain a conclusive answer without a controlled, prospective study because of the almost universal use of potent ICS in moderate to severe asthma. In any case any risk of increased colonisation, in asthma at least, is outweighed by the beneficial effects of ICS in AFAD. Although clearly linked, the precise relationship between sensitisation and colonisation remains uncertain. ${ }^{102}$

\section{Clinical Features of AFAD}

AFAD is diagnosed by the identification of specific $\operatorname{IgE}$ against A. fumigatus and related thermotolerant fungi (a Penicillium species should be in the panel), in the context of airway disease, although this is not always apparent at presentation. $^{15,103}$ (Table 3). Sensitisation can be detected

\section{Table 3}

\begin{tabular}{|l|}
\hline Presentations of Allergic Fungal Airway Disease \\
\hline $\begin{array}{l}\text { Difficult-to-manage asthma (severe asthma with fungal sensitisation } \\
(\mathrm{SAFS}) \text { ) and less commonly COPD }\end{array}$ \\
\hline Allergic bronchopulmonary aspergillosis/mycosis (ABPA/M) \\
\hline Chronic eosinophilic pneumonia \\
\hline Lobar collapse \\
\hline Fixed airflow obstruction in later life \\
\hline Bronchiectasis and upper lobe fibrosis \\
\hline Persistent sputum production (fungal bronchitis) \\
\hline $\begin{array}{l}\text { Unexplained marked peripheral blood eosinophilia or cause of total } \\
\text { IgE > I000 IU/L }\end{array}$ \\
\hline Fungal empyema \\
\hline
\end{tabular}


by skin prick testing against fungal extracts, using a cutoff of $3 \mathrm{~mm}$ equal to or greater than the diluent, or in vitro laboratory testing. A positive specific $\operatorname{IgE}$ is best interpreted qualitatively rather than quantitatively as it appears that even low levels of specific $\mathrm{IgE}$ are clinically relevant. Indeed it is likely that in some cases concentrations below the $0.35 \mathrm{IU} / \mathrm{L}$ cut-off often used for the ImmunoCap assay may be diagnostic of AFAD. In this setting analysis of the commercially available allergen components Asp f1-4 and Asp f6 may reveal a more clear-cut positive result. In our experience skin tests, although generally quite specific, are less sensitive than measurement of specific IgE, with only $60 \%$ of positive tests with the latter also positive with the former technique (AJW personal observation). ${ }^{104}$

\section{Asthma}

Approximately one-third of patients attending difficult asthma clinics have AFAD complicating their asthma. ${ }^{105}$ The characteristic features specific to allergy to colonising, thermotolerant filamentous fungi are those of lung damage (bronchiectasis, which is more often distal than central, FAO and lung fibrosis). ${ }^{106,107}$ Fleeting shadows are also characteristic, though rarely seen in the modern era of widespread use of potent ICS. Airway plugging with hyperdense mucus which is associated with tree-in-bud shadowing are other characteristic features seen in highresolution CAT (HRCT) scans. ${ }^{108-110}$ Exacerbations in AFAD are not especially common compared to the often adult onset, "hyper-eosinophilic" phenotype, although in a Singaporean cohort where only $12 \%$ of severe asthmatics were sensitised to A. fumigatus there was an association with severe exacerbations. ${ }^{8,111,112}$ Where they occur, exacerbations are often driven by bacterial or fungal bronchitis rather than eosinophilic inflammation, especially in those patients with bronchiectasis. ${ }^{5}$ We analysed a large cohort of patients with moderate to severe asthma enriched for AFAD. ${ }^{20}$ The patients with AFAD had higher rates of early-onset disease and as a result almost twice the duration of asthma. They were twice as likely to be atopic but in other respects were similar to their non-fungal sensitised counterparts. As with our original report those with AFAD had overall about a $10 \%$ deficit in $\mathrm{FEV}_{1}$ which was not related to atopy and not seen in patients sensitised to nonthermotolerant or non-filamentous fungi. Significant differences in radiological appearances between those sensitised and non-sensitised to fungi included bronchiectasis (50\% versus $29 \%$ ), tree-in-bud (17\% vs $4 \%$ ) and collapse/ consolidation ( $35 \%$ vs $21 \%)$. Fleeting shadows just missed significance as they were only seen in $4.5 \%$ vs $0 \%$ of cases. Hyper-attenuated mucus was not commented on in the radiology report, but often occurs as part of tree-in-bud shadowing. Fibrosis occurred more commonly $(11 \%$ vs $5 \%$ ), but this did not reach significance. As with lung function these abnormalities were only related to $\operatorname{IgE}$ sensitisation to thermotolerant filamentous fungi. A positive IgE to A. fumigatus was related to all the radiological abnormalities, but the total $\operatorname{IgE}$ was only related to tree-in-bud shadowing and fleeting shadows, with only the latter statistically associated with a total IgE of $>1000 \mathrm{IU} / \mathrm{L}$. Interestingly the A. fumigatus $\mathrm{IgG}$ was, albeit relatively weakly, associated with all the radiological abnormalities except collapse/consolidation, but at levels below the accepted normal cut-off of $40 \mu \mathrm{g} /$ L. A K-means cluster analysis of the cohort picked out $\sim 8 \%$ of subjects with high concentrations of specific and total IgE and elevated rates of lung damage. This group presumably consists of those being recognised as having ABPA, although equal numbers of subjects who met the ISHAM criteria for ABPA were present in another cluster without these features.

\section{Cystic Fibrosis (CF)}

Rates of IgE sensitisation to A. fumigatus (and a positive sputum fungal culture) in CF are similar to those in severe asthma, and there has been a related debate regarding the criteria for ABPA with the extensive lung damage which is the hallmark of this condition making it even more difficult than in asthma and COPD to separate the underlying disease from the effects of fungal allergy. As with asthma the criteria for diagnosis of ABPA are often applied flexibly. ${ }^{113}$ Sensitisation to A. fumigatus has also been found to be associated with accelerated lung function decline in cystic fibrosis. ${ }^{114}$ The A. fumigatus $\operatorname{IgG}$ is often high in $\mathrm{CF}$, suggesting a more consistently raised fungal load in the lungs compared to asthma.

\section{Other Presentations of AFAD}

In our specialist clinic for patients with AFAD we encountered a number of less common presentations. Eosinophilic pneumonia with extensive lung shadowing associated with a marked BAL and blood eosinophilia, which is highly glucocorticoid sensitive, is a well-recognised complication of AFAD and has also been described in the context of $\operatorname{IgE}$ sensitisation to C. albicans. ${ }^{15,116}$ A large dose of inhaled A. fumigatus spores, for example in the context of gardening or indoor contamination, can sometimes be identified 
as a trigger. Similarly, lobar collapse due to large-airway obstruction with inspissated mucus is a classical if unusual presentation. It is often diagnosed initially as lung cancer if the invariable peripheral blood eosinophilia is missed, only being recognised at bronchoscopy, and sometimes not even then. ${ }^{117}$ Non-smoking fixed airflow obstruction presenting in late middle age can be caused by AFAD, usually, but not always with a long-standing history of relatively mild asthma. These patients often attract a diagnosis of COPD (a diagnosis best reserved for people with a heavy smoking history). Allergy to both skin- and airway-colonising yeasts and filamentous fungi is a cause of a marked peripheral blood eosinophilia and $\operatorname{IgE}>1000$ IU/L, and patients with mild AFAD where the airway disease has not been recognised can be referred because of concern about these findings. ${ }^{118}$ Usually the immunological picture in chronic pulmonary aspergillosis is quite different to AFAD with a neutrophilia, no IgE sensitisation, a low total IgE and a very high specific IgG. Occasionally, however, they overlap, and AFAD exists alongside a clinical picture of upper lobe cavitation, fungal pneumonia or empyema. ${ }^{119}$

\section{Fungal Bronchitis and the Role of Anti-Fungal Therapy in AFAD}

As the pathogenesis of AFAD is thought to include the germination of fungal spores in the airway, it would be rational to consider using anti-fungal therapy to eradicate the fungi. However, the evidence that anti-fungal treatments are sufficiently effective for routine use in AFAD is limited, and their use is further limited by cost, adverse events, resistance and failure to achieve satisfactory tissue concentrations. $^{120}$ In most cases, triazole anti-fungal agents, that prevent conversion of lanosterol to ergosterol by inhibition of lanosterol $14 \alpha$-demethylase, are first-line therapy, with echinocandins, that inhibit the synthesis of $\beta$ glucan in the fungal cell wall, as second-line agents. However, echinocandins can only be given intravenously and have significant adverse events such as nephrotoxicity and hepatotoxicity, which limits their use. The most commonly used triazole, mainly because of its relatively low cost, is itraconazole. Alternatives are voriconazole, posaconazole and the recently introduced isavuconazole. These all have some advantages in terms of tissue penetration, tolerability and efficacy, but they are more expensive. Although they can be helpful if there is itraconazole resistance, often this is the result of mutations in the fungus that cause pan-triazole resistance. The major side effects which result in having to stop the drugs are gastric intolerance, hepatotoxicity, peripheral neuropathy and hair loss which can be permanent. They also cause photosensitivity and can be cardiotoxic so are relatively contraindicated in people with significant congestive cardiac failure. Itraconazole causes a feeling of intolerable malaise in some individuals as well as enhancing bioavailability of natural and exogenous glucocorticoids, resulting in the risk of an adrenal crisis after a prolonged course is stopped, as well as sometimes complicating interpretation of benefit. As well as adverse events, they induce liver enzymes and as a result interact with a lot of other medications, which complicates and sometimes limits their use. The variable absorption of these drugs through the GI mucosa means that it is essential to measure trough blood concentrations one week after starting treatment and adjust the dose accordingly. This also helps to reduce the risk of side effects. It is also important to measure sensitivity to the triazoles as resistance is not uncommon, particularly if the patient has previously been treated with a triazole, and this can lead to treatment failure. Treatment courses are much more prolonged than for anti-bacterial medications. There are no definitive guidelines on how long a course should be, but in our experience three months is necessary and usually sufficient, although if there is going to be a response there is usually some evidence for this at one month and improvement can continue for some time after stopping. Repeated courses are sometimes necessary. It is often the case that one triazole works where another one does not, often for no obvious reason. Our practice is to start with itraconazole, assuming the fungal species in question is sensitive, but change to either posaconazole or voriconazole after one month if there has not been any improvement despite good blood levels. Anti-fungal treatment is not therefore for the faint-hearted or casual prescriber, especially considering the comments below about efficacy in AFAD. The patients need to be carefully selected both in terms of the extent to which their symptoms are likely to be due to infection rather than allergic inflammation, and in terms of the risk of adverse events. Treatment needs to be carried out with the help of a microbiologist and pharmacist with an interest in fungal disease with appropriate patient advice and monitoring. The above comments make it clear that there is room for improvement in the anti-fungal drugs available to treat AFAD. The obvious way forward would be an inhaled triazole that would generate high concentrations of the 
drug in the lungs without the risk of side effects due to high plasma concentrations. There are no such licensed preparations. Lipophilic amphotericin has been used as a nebulised therapy but is poorly tolerated. There is also an emerging literature on dry powder formulations for inhalation of itraconazole and voriconazole. Particularly promising is a novel potent triazole PC945 being developed by a small biotech company (Pulmocide) specifically for airway delivery. ${ }^{121,122}$

There have been four fully randomised and placebocontrolled studies of anti-fungal agents in AFAD, all of which were small. ${ }^{21,123-125}$ Two used itraconazole in patients labelled as having ABPA, one used itraconazole in SAFS and one used voriconazole in AFAD, although in practice the patients were all similar. The end points and study designs were varied. Stevens et al used a complicated clinical score to assess a modest improvement, the work by Wark et al was designed to study inflammatory changes rather than clinical improvement, Denning et al found isolated improvement in quality of life after several months of treatment and Agbetile et al found no improvement in any of the parameters investigated. The interpretation of the itraconazole studies in particular are complicated by the known marked corticosteroid-enhancing property of this drug. In contrast there are several case series/reports and uncontrolled trials suggesting an improvement in symptoms and outcomes with triazole anti-fungal therapy. ${ }^{126,127}$ We observed that two or three subjects in our trial of 32 voriconazole-treated patients with AFAD associated with asthma appeared to gain a clear benefit. These subjects each had a productive cough and grew large amounts of A. fumigatus in their sputum. This led us to propose the hypothesis that antifungal agents may not have a role in treating the inflammatory aspects of AFAD, but would be effective in patients with fungal bronchitis. ${ }^{128,129}$ We have defined this term as representing exacerbations of airway disease, characterised by a chronic cough productive of highly viscous or even rubbery brown or creamy sputum, unresponsive to broad-spectrum antibiotics and high-dose oral glucocorticoids, with a positive sputum culture for a relevant fungus (usually A. fumigatus or Candida spp.). We have found that patients with this presentation almost invariably improve with triazole therapy, often quite dramatically, both in terms of symptoms and lung function, with improvement (if it is going to occur) obvious within one month of treatment. ${ }^{5}$ A. fumigatus bronchitis usually occurred in patients with bronchiectasis associated with
AFAD, but this was less often the case with Candida bronchitis. As noted above, an inhaled form of these drugs would be a great advance in the treatment of fungal bronchitis, a disease which goes largely unrecognised and is probably quite common as a complication of both asthma and COPD.

\section{AFAD in Children}

Most patients with AFAD present after the fourth decade, and ABPA was regarded as rare in childhood in association with asthma, although it is well recognised in cystic fibrosis. ${ }^{130,131}$ Fungal allergy is, however, a feature of severe asthma in children, and it is reasonable to assume that the sensitisation and possibly the damaging effects of AFAD start at this age. ${ }^{132}$ Castanhinha et al found increased IL-33 expression in the airways of children with SAFS compared to non-fungal sensitised patients with therapy-resistant asthma, although they did not report how many were only sensitised to A. fumigatus rather than Alternaria and Cladosporium spp. ${ }^{133}$ In a cohort of 175 children consisting of chronic asthmatics attending a secondary care asthma clinic, acute asthmatics admitted with an exacerbation and healthy controls, nearly $50 \%$ of the asthmatics were IgE-sensitised to fungal allergens with $34 \%$ sensitised to thermotolerant fungi. This compared with no healthy children sensitised to fungi despite $27 \%$ being atopic. Children with fungal sensitisation had evidence of more severe disease including worse lung function, demonstrating that the close link between fungal allergy and severe asthma seen in adults also holds true in children. ${ }^{134}$

\section{Management of AFAD}

The management of AFAD is by and large similar to that of the underlying airway disease, with the exception that sources of fungal exposure should be explored and fungal bronchitis should be looked for in case anti-fungal therapy is warranted. Otherwise, inhaled corticosteroids and bronchodilators are effective where asthma and to a lesser extent COPD are present, and omalizumab and anti-IL-5 biological therapy appears to be at least equally effective in those with more severe exacerbation-prone disease. $^{22,80}$ The high eosinophil and IgE levels that are such a feature of fungal allergy are not of themselves a cause for concern. They do not track particularly closely with disease severity or activity, and there is little evidence that the often marked peripheral blood eosinophilia causes the multi-organ damage seen with hypereosinophilic 
syndrome. ${ }^{118}$ As AFAD often causes severe disease, it used to be common that patients required long-term oral corticosteroids, but with the advent of anti-T2 biological therapies that may not be such a problem in the future. Once lung damage has become established, patients are more difficult to manage, with chronic breathlessness due to FAO a particular problem, and bacterial bronchitis (as well as fungal bronchitis) a common occurrence. The former may be helped by pulmonary rehabilitation and the latter by broad-spectrum antibiotics and lung physiotherapy.

\section{Summary}

Allergy to colonising, filamentous, thermotolerant fungi represents a common (in secondary care) endotype of airway diseases such as asthma, COPD, cystic fibrosis and primary bronchiectasis. Pathogenesis is likely to be primarily due to a vigorous, persistent, $\mathrm{T} 2$ immune response to germinating airway fungi leading to marked eosinophilic inflammation, with resultant production of highly viscous mucus. Less commonly it can present de novo without a previous diagnosis of underlying lung disease. The heterogeneous presentation of this endotype means that under current knowledge, with limited biomarkers of disease severity, we propose that it is best described using inclusive criteria represented by the term allergic fungal airways disease, rather than the exclusive SAFS or ABPA. Lung damage, which is the hallmark of AFAD, occurs over many decades, usually presenting in the second half of life. Less common presentations include lobar collapse and eosinophilic pneumonia. Management in sensitised individuals should be aimed, firstly, at reducing fungal exposure, for example by addressing areas of dampness indoors, identifying and managing potential fungal exposure in the workplace and limiting exposure outdoors particularly in the context of gardening by limiting contact with decaying vegetation and wearing appropriate masks. Secondly, prevention of exacerbations, which probably accelerate the development of lung damage, is important by controlling eosinophilic inflammation with glucocorticoids and if necessary anti-T2 biological therapy. Once bronchiectasis is present it can cause recurrent episodes of bacterial and fungal bronchitis, the latter effectively treated with triazole anti-fungal therapy, which otherwise does not have a place in the management of AFAD. The use of such therapy is, however, not straightforward for reasons of adverse events, drug interactions, cost, resistance and poor absorption, so the development of bespoke nebulised antifungal treatment, which could obviate at least some of these difficulties, would be a significant advance. Chronic breathlessness caused by impaired lung function may benefit from pulmonary rehabilitation. It is likely that the progression to severe disease starts in childhood so that recognition and close observation of children with IgE sensitisation to A. fumigatus and related fungi, who in any case are likely to have more difficult-to-control airway disease, is warranted.

\section{Abbreviations}

ABPA/M, allergic bronchopulmonary aspergillosis/mycosis; AFAD, allergic fungal airway disease; AFRS, allergic fungal rhinosinusitis; FAO, fixed airflow obstruction; G\&C, Gell and Coombs; ICS, inhaled corticosteroids; SAFS, severe asthma with fungal sensitisation.

\section{Acknowledgments}

This study was supported by the NIHR Leicester Biomedical Research Centre. The views expressed are those of the authors and not necessarily those of the NHS, the NIHR or the Department of Health.

\section{Disclosure}

AJW has received funding for advisory work from GSK and Pulmocide and grants for research from GSK, AstraZeneca and Pulmocide. He holds $£ 1000$ in GSK shares. EAG reports personal fees from Boehringer Ingelheim, grants from Circassia, non-financial support from Medimmune, outside the submitted work. CHP has received funding for her research from Pulmocide. The authors report no other conflicts of interest in this work.

\section{References}

1. Denning DW, O’Driscoll BR, Hogaboam CM, Bowyer P, Niven RM. The link between fungi and severe asthma: a summary of the evidence. Eur Respir J. 2006;27(3):615-626. doi:10.1183/ 09031936.06.00074705

2. Denning DW, Pashley C, Hartl D, et al. Fungal allergy in asthma-state of the art and research needs. Clin Transl Allerg. 2014;4:14. doi:10.1186/2045-7022-4-14

3. Knutsen AP, Bush RK, Demain JG, et al. Fungi and allergic lower respiratory tract diseases. $J$ Allergy Clin Immunol. 2012;129 (2):280-283. doi:10.1016/j.jaci.2011.12.970

4. Wardlaw AJ, Woolnough K, Pashley $\mathrm{CH}$. Lassoing a chimera: the semantics of allergic fungal airway disease. Clin Exp Allergy. 2015;45(12):1746-1749. doi:10.1111/cea.12659

5. Ozyigit LP, Monteiro W, Rick EM, Satchwell J, Pashley CH, Wardlaw AJ. Fungal bronchitis is a distinct clinical entity which is responsive to antifungal therapy. Chronic Respir Dis. 2021;18:1479973120964448. doi:10.1177/1479973120964448 
6. Pavord ID, Wardlaw AJ. The A to E of airway disease. Clin Exp Allergy. 2010;40(1):62-67. doi:10.1111/j.1365-2222.2009.03410.x

7. Wardlaw AJ, Silverman M, Siva R, Pavord ID, Green R. Multidimensional phenotyping: towards a new taxonomy for airway disease. Clin Exp Allergy. 2005;35(10):1254-1262. doi:10.1111/ j.1365-2222.2005.02344.x

8. Haldar P, Pavord ID, Shaw DE, et al. Cluster analysis and clinical asthma phenotypes. Am J Respir Crit Care Med. 2008;178 (3):218-224. doi:10.1164/rccm.200711-1754OC

9. Anderson GP. Endotyping asthma: new insights into key pathogenic mechanisms in a complex, heterogeneous disease. Lancet. 2008;372(9643):1107-1119. doi:10.1016/S0140-6736(08)61452-X

10. Rosenberg M, Patterson R, Mintzer R, Cooper BJ, Roberts M, Harris KE. Clinical and immunologic criteria for the diagnosis of allergic bronchopulmonary aspergillosis. Ann Intern Med. 1977;86(4):405-414. doi:10.7326/0003-4819-86-4-405

11. Hinson KF, Moon AJ, Plummer NS. Broncho-pulmonary aspergillosis; a review and a report of eight new cases. Thorax. 1952;7 (4):317-333. doi:10.1136/thx.7.4.317

12. Greenberger PA, Bush RK, Demain JG, Luong A, Slavin RG, Knutsen AP. Allergic bronchopulmonary aspergillosis. J Allergy Clin Immunol Pract. 2014;2(6):703-708. doi:10.1016/j. jaip.2014.08.007

13. Agarwal R, Maskey D, Aggarwal AN, et al. Diagnostic performance of various tests and criteria employed in allergic bronchopulmonary aspergillosis: a latent class analysis. PLoS One. 2013;8(4):e61105. doi:10.1371/journal.pone.0061105

14. Asano K, Hebisawa A, Ishiguro T, et al. New clinical diagnostic criteria for allergic bronchopulmonary aspergillosis/mycosis and its validation. $J$ Allergy Clin Immunol. 2020;147:1261-1268.e5. doi:10.1016/j.jaci.2020.08.029

15. Li E, Landers CT, Tung HY, et al. Fungi in mucoobstructive airway diseases. Ann Am Thorac Soc. 2018;15(Suppl 3):S198S204. doi:10.1513/AnnalsATS.201803-154AW

16. Tiew PY, Ko FWS, Pang SL, et al. Environmental fungal sensitisation associates with poorer clinical outcomes in COPD. Eur Respir J. 2020;56(2):2000418. doi:10.1183/13993003.00418-2020

17. Bafadhel M, McKenna S, Agbetile J, et al. Aspergillus fumigatus during stable state and exacerbations of COPD. Eur Respir J. 2013;43:64-71. doi:10.1183/09031936.00162912

18. Everaerts S, Lagrou K, Dubbeldam A, et al. Sensitization to Aspergillus fumigatus as a risk factor for bronchiectasis in COPD. Int J Chron Obstruct Pulmon Dis. 2017;12:2629-2638. doi:10.2147/COPD.S141695

19. Sehgal IS, Dhooria S, Prasad KT, et al. Sensitization to A fumigatus in subjects with non-cystic fibrosis bronchiectasis. Mycoses. 2020.

20. Woolnough KF, Richardson M, Newby C, et al. The relationship between biomarkers of fungal allergy and lung damage in asthma. Clin Exp Allergy. 2017;47(1):48-56. doi:10.1111/cea.12848

21. Denning DW, O'Driscoll BR, Powell G, et al. Randomized controlled trial of oral antifungal treatment for severe asthma with fungal sensitization: the Fungal Asthma Sensitization Trial (FAST) study. Am J Respir Crit Care Med. 2009;179(1):11-18. doi:10.1164/rccm.200805-737OC

22. Wark P, Hussaini S, Holder C, Powell H, Gibson P, Oldmeadow C. Omalizumab is an effective intervention in severe asthma with fungal sensitization. $J$ Allergy Clin Immunol Pract. 2020;8(10):3428-33 e1. doi:10.1016/j.jaip.2020.05.055

23. Blakey JD, Wardlaw AJ. What is severe asthma? Clin Exp Allergy. 2012;42(5):617-624. doi:10.1111/j.13652222.2012.03962.x

24. Lotvall J, Akdis CA, Bacharier LB, et al. Asthma endotypes: a new approach to classification of disease entities within the asthma syndrome. $J$ Allergy Clin Immunol. 2011;127 (2):355-360. doi:10.1016/j.jaci.2010.11.037
25. Tyler MA, Luong AU. Current concepts in the management of allergic fungal rhinosinusitis. Immunol Allergy Clin North Am. 2020;40(2):345-359. doi:10.1016/j.iac.2019.12.001

26. Bent JP, Kuhn FA. Diagnosis of allergic fungal sinusitis. Otolaryngol Head Neck Surg. 1994;111(5):580-588. doi: $10.1177 / 019459989411100508$

27. Hawksworth DL, Lucking R. Fungal diversity revisited: 2.2 to 3.8 million species. Microbiol Spectr. 2017;5(4). doi:10.1128/ microbiolspec.FUNK-0052-2016

28. Pashley CH, Fairs A, Free RC, Wardlaw AJ. DNA analysis of outdoor air reveals a high degree of fungal diversity, temporal variability, and genera not seen by spore morphology. Fungal Biol. 2012;116(2):214-224. doi:10.1016/j.funbio.2011.11.004

29. Simon-Nobbe B, Denk U, Poll V, Rid R, Breitenbach M. The spectrum of fungal allergy. Int Arch Allergy Immunol. 2008;145 (1):58-86. doi:10.1159/000107578

30. Crameri R, Garbani M, Rhyner C, Huitema C. Fungi: the neglected allergenic sources. Allergy. 2014;69(2):176-185. doi:10.1111/all.12325

31. Pedrosa AF, Lisboa C, Rodrigues AG. Malassezia infections with systemic involvement: figures and facts. $J$ Dermatol. 2018;45 (11):1278-1282. doi:10.1111/1346-8138.14653

32. Petrucelli MF, Abreu MH, Cantelli BAM, et al. Epidemiology and diagnostic perspectives of dermatophytoses. J Fungi (Basel). 2020;6(4). doi:10.3390/jof6040310.

33. Woodfolk JA. Allergy and dermatophytes. Clin Microbiol Rev. 2005;18(1):30-43. doi:10.1128/CMR.18.1.30-43.2005

34. Adav SS, Ravindran A, Sze SK. Proteomic analysis of temperature dependent extracellular proteins from Aspergillus fumigatus grown under solid-state culture condition. J Proteome Res. 2013;12(6):2715-2731. doi:10.1021/pr4000762

35. Douglas P, Tyrrel SF, Kinnersley RP, et al. Predicting Aspergillus fumigatus exposure from composting facilities using a dispersion model: a conditional calibration and validation. Int $J$ Hyg Environ Health. 2017;220(1):17-28. doi:10.1016/j.ijheh.2016.09.017

36. Fairs A, Wardlaw AJ, Thompson JR, Pashley CH. Guidelines on ambient intramural airborne fungal spores. $J$ Investig Allergol Clin Immunol. 2010;20(6):490-498.

37. Andersen B, Frisvad JC, Sondergaard I, Rasmussen IS, Larsen LS. Associations between fungal species and water-damaged building materials. Appl Environ Microbiol. 2011;77(12):4180-4188. doi:10.1128/AEM.02513-10

38. Aimanianda V, Latge JP. Fungal hydrophobins form a sheath preventing immune recognition of airborne conidia. Virulence. 2010;1(3):185-187. doi:10.4161/viru.1.3.11317

39. Barnes C. Fungi and atopy. Clin Rev Allergy Immunol. 2019;57 (3):439-448. doi:10.1007/s12016-019-08750-z

40. Douwes J, Thorne P, Pearce N, Heederik D. Bioaerosol health effects and exposure assessment: progress and prospects. Ann Occup Hyg. 2003;47(3):187-200. doi:10.1093/annhyg/meg032

41. Quirce S, Vandenplas O, Campo P, et al. Occupational hypersensitivity pneumonitis: an EAACI position paper. Allergy. 2016;71 (6):765-779. doi:10.1111/all.12866

42. Barbeau DN, Grimsley LF, White LE, El-Dahr JM, Lichtveld M. Mold exposure and health effects following hurricanes Katrina and Rita. Annu Rev Public Health. 2010;31:165-78 1 p following 78. doi:10.1146/annurev.publhealth.012809.103643

43. Oluyomi AO, Panthagani K, Sotelo J, et al. Houston hurricane Harvey health (Houston-3H) study: assessment of allergic symptoms and stress after hurricane Harvey flooding. Environ Health. 2021;20(1):9. doi:10.1186/s12940-021-00694-2

44. Tischer C, Chen CM, Heinrich J. Association between domestic mould and mould components, and asthma and allergy in children: a systematic review. Eur Respir J. 2011;38(4):812-824. doi:10.1183/09031936.00184010 
45. Fukutomi Y, Taniguchi M. Sensitization to fungal allergens: resolved and unresolved issues. Allergol Int. 2015;64 (4):321-331. doi:10.1016/j.alit.2015.05.007

46. Goodman RE, Chapman MD, Slater JE. The allergen: sources, extracts, and molecules for diagnosis of allergic disease. J Allergy Clin Immunol Pract. 2020;8(8):2506-2514. doi:10.1016/j. jaip.2020.06.043

47. Fukutomi Y, Tanimoto H, Yasueda H, Taniguchi M. Serological diagnosis of allergic bronchopulmonary mycosis: progress and challenges. Allergol Int. 2016;65(1):30-36. doi:10.1016/j. alit.2015.08.004

48. Crameri R. The problem of cross-reactivity in the diagnosis of fungal allergy. Clin Exp Allergy. 2011;41(3):302-304. doi:10.1111/j.1365-2222.2011.03703.x

49. Woolnough K, Craner M, Pashley CH, Wardlaw AJ. rAsp f3 and rAsp $\mathrm{f} 4$ are associated with bronchiectasis in allergic fungal airways disease. Ann Allergy Asthma Immunol. 2018;120 (3):325-326. doi:10.1016/j.anai.2017.10.026

50. Database WA. Allergen Nomenclature; 2020. Available from: http://www.allergen.org/search.php?allergensource=Aspergillus +fumigatus. Accessed May 10, 2021.

51. Tanimoto H, Fukutomi Y, Yasueda H, et al. Molecular-based allergy diagnosis of allergic bronchopulmonary aspergillosis in Aspergillus fumigatus-sensitized Japanese patients. Clin Exp Allergy. 2015;45(12):1790-1800. doi:10.1111/cea.12590

52. Muthu V, Sehgal IS, Dhooria S, Aggarwal AN, Agarwal R. Utility of recombinant Aspergillus fumigatus antigens in the diagnosis of allergic bronchopulmonary aspergillosis: a systematic review and diagnostic test accuracy meta-analysis. Clin Exp Allergy. 2018;48(9):1107-1136. doi:10.1111/cea.13216

53. Russell RJ, Brightling C. Pathogenesis of asthma: implications for precision medicine. Clin Sci (Lond). 2017;131 (14):1723-1735. doi:10.1042/CS20160253

54. Boonpiyathad T, Sozener ZC, Satitsuksanoa P, Akdis CA. Immunologic mechanisms in asthma. Semin Immunol. 2019;46:101333. doi:10.1016/j.smim.2019.101333

55. Mackel JJ, Steele C. Host defense mechanisms against Aspergillus fumigatus lung colonization and invasion. Curr Opin Microbiol. 2019;52:14-19.

56. Bacher P, Hohnstein T, Beerbaum E, et al. Human anti-fungal Th17 immunity and pathology rely on cross-reactivity against Candida albicans. Cell. 2019;176(6):1340-55 e15. doi:10.1016/j. cell.2019.01.041

57. Choy DF, Hart KM, Borthwick LA, et al. TH2 and TH17 inflammatory pathways are reciprocally regulated in asthma. Sci Transl Med. 2015;7(301):301ra129. doi:10.1126/scitranslmed.aab3142

58. Siddiqui S, Shikotra A, Richardson M, et al. Airway pathological heterogeneity in asthma: visualization of disease microclusters using topological data analysis. $J$ Allergy Clin Immunol. 2018;142(5):1457-1468. doi:10.1016/j.jaci.2017.12.982

59. Ueki S, Hebisawa A, Kitani M, Asano K, Neves JS. Allergic bronchopulmonary aspergillosis-A luminal hypereosinophilic disease with extracellular trap cell death. Front Immunol. 2018;9:2346. doi:10.3389/fimmu.2018.02346

60. Yike I. Fungal proteases and their pathophysiological effects. Mycopathologia. 2011;171(5):299-323. doi:10.1007/s11046-0109386-2

61. Wartenberg D, Lapp K, Jacobsen ID, et al. Secretome analysis of Aspergillus fumigatus reveals Asp-hemolysin as a major secreted protein. Int J Med Microbiol. 2011;301(7):602-611. doi:10.1016/ j.ijmm.2011.04.016

62. Druey KM, McCullough M, Krishnan R. Aspergillus fumigatus protease alkaline protease 1 (Alp1): a new therapeutic target for fungal asthma. J Fungi (Basel). 2020;6(2). doi:10.3390/ jof6020088
63. Wiesner DL, Merkhofer RM, Ober C, et al. Club cell TRPV4 serves as a damage sensor driving lung allergic inflammation. Cell Host Microbe. 2020;27(4):614-28 e6. doi:10.1016/j. chom.2020.02.006

64. Balenga NA, Klichinsky M, Xie Z, et al. A fungal protease allergen provokes airway hyper-responsiveness in asthma. Nat Commun. 2015;6:6763. doi:10.1038/ncomms7763

65. Basu T, Seyedmousavi S, Sugui JA, et al. Aspergillus fumigatus alkaline protease 1 (Alp1/Asp f13) in the airways correlates with asthma severity. J Allergy Clin Immunol. 2018;141(1):423-5 e7. doi:10.1016/j.jaci.2017.07.034

66. Agarwal R, Khan A, Gupta D, Aggarwal AN, Saxena AK, Chakrabarti A. An alternate method of classifying allergic bronchopulmonary aspergillosis based on high-attenuation mucus. PLoS One. 2010;5(12):e15346. doi:10.1371/journal. pone.0015346

67. Lu HW, Mao B, Wei P, et al. The clinical characteristics and prognosis of ABPA are closely related to the mucus plugs in central bronchiectasis. Clin Respir J. 2020;14(2):140-147. doi:10.1111/crj.13111

68. Bonser LR, Erle DJ. Airway mucus and asthma: the role of MUC5AC and MUC5B. J Clin Med. 2017;6(12):112. doi:10.3390/jcm6120112

69. Dunican EM, Watchorn DC, Fahy JV. Autopsy and imaging studies of mucus in asthma. Lessons learned about disease mechanisms and the role of mucus in airflow obstruction. Ann Am Thorac Soc. 2018;15(Suppl 3):S184-S91. doi:10.1513/ AnnalsATS.201807-485AW

70. Dunican EM, Elicker BM, Gierada DS, et al. Mucus plugs in patients with asthma linked to eosinophilia and airflow obstruction. J Clin Invest. 2018;128(3):997-1009. doi:10.1172/ JCI95693

71. Dournes G, Berger P, Refait J, et al. Allergic bronchopulmonary aspergillosis in cystic fibrosis: MR imaging of airway mucus contrasts as a tool for diagnosis. Radiology. 2017;285 (1):261-269. doi:10.1148/radiol.2017162350

72. Boyton RJ, Altmann DM. Bronchiectasis: current concepts in pathogenesis, immunology, and microbiology. Annu Rev Pathol. 2016;11:523-554. doi:10.1146/annurev-pathol-012615-044344

73. Bush A, Floto RA. Pathophysiology, causes and genetics of paediatric and adult bronchiectasis. Respirology. 2019;24 (11):1053-1062. doi:10.1111/resp.13509

74. Cole PJ. Inflammation: a two-edged sword-the model of bronchiectasis. Eur J Respir Dis Suppl. 1986;147:6-15.

75. Li E, Knight JM, Wu Y, et al. Airway mycosis in allergic airway disease. Adv Immunol. 2019;142:85-140.

76. Burgel PR, Lazarus SC, Tam DC, et al. Human eosinophils induce mucin production in airway epithelial cells via epidermal growth factor receptor activation. $J$ Immunol. 2001;167 (10):5948-5954. doi:10.4049/jimmunol.167.10.5948

77. Persson EK, Verstraete K, Heyndrickx I, et al. Protein crystallization promotes type 2 immunity and is reversible by antibody treatment. Science. 2019;364:6442. doi:10.1126/science. aaw4295

78. Muniz VS, Silva JC, Braga YAV, et al. Eosinophils release extracellular DNA traps in response to Aspergillus fumigatus. $J$ Allergy Clin Immunol. 2018;141(2):571-85 e7. doi:10.1016/j. jaci.2017.07.048

79. Figueiredo RT, Neves JS. Eosinophils in fungal diseases: an overview. J Leukoc Biol. 2018;104(1):49-60. doi:10.1002/ JLB.4MR1117-473R

80. Wardlaw A, Howarth PH, Israel E, et al. Fungal sensitization and its relationship to mepolizumab response in patients with severe eosinophilic asthma. Clin Exp Allergy. 2020;50(7):869-872. doi: $10.1111 /$ cea. 13680 
81. Fabbri LM, Romagnoli M, Corbetta L, et al. Differences in airway inflammation in patients with fixed airflow obstruction due to asthma or chronic obstructive pulmonary disease. Am J Respir Crit Care Med. 2003;167(3):418-424. doi:10.1164/rccm.200203-183OC

82. Hirahara K, Aoki A, Morimoto Y, Kiuchi M, Okano M, Nakayama T. The immunopathology of lung fibrosis: amphiregulin-producing pathogenic memory $\mathrm{T}$ helper-2 cells control the airway fibrotic responses by inducing eosinophils to secrete osteopontin. Semin Immunopathol. 2019;41(3):339-348. doi:10.1007/s00281-019-00735-6

83. Nhu QM, Aceves SS. Tissue remodeling in chronic eosinophilic esophageal inflammation: parallels in asthma and therapeutic perspectives. Front Med (Lausanne). 2017;4:128. doi:10.3389/ fmed.2017.00128

84. Gieseck RL, Wilson MS, Wynn TA. Type 2 immunity in tissue repair and fibrosis. Nat Rev Immunol. 2018;18(1):62-76.

85. Bartemes KR, Kita H. Innate and adaptive immune responses to fungi in the airway. $J$ Allergy Clin Immunol. 2018;142 (2):353-363. doi:10.1016/j.jaci.2018.06.015

86. Chauhan B, Hutcheson PS, Slavin RG, Bellone CJ. MHC restriction in allergic bronchopulmonary aspergillosis. Front Biosci. 2003;8:s140-8. doi:10.2741/971

87. Agarwal R, Chakrabarti A, Shah A, et al. Allergic bronchopulmonary aspergillosis: review of literature and proposal of new diagnostic and classification criteria. Clin Exp Allergy. 2013;43 (8):850-873.

88. Pashley CH, Fairs A, Morley JP, et al. Routine processing procedures for isolating filamentous fungi from respiratory sputum samples may underestimate fungal prevalence. Med Mycol. 2012;50(4):433-438. doi:10.3109/13693786.2011.615762

89. Vergidis P, Moore CB, Novak-Frazer L, et al. High-volume culture and quantitative real-time PCR for the detection of Aspergillus in sputum. Clin Microbiol Infect. 2020;26 (7):935-940. doi:10.1016/j.cmi.2019.11.019

90. Pappas PG, Kauffman CA, Andes DR, et al. Executive summary: clinical practice guideline for the management of candidiasis: 2016 update by the Infectious Diseases Society of America. Clin Infect Dis. 2016;62(4):409-417. doi:10.1093/cid/civ1194

91. Agbetile J, Fairs A, Desai D, et al. Isolation of filamentous fungi from sputum in asthma is associated with reduced post-bronchodilator FEV1. Clin Exp Allergy. 2012;42 (5):782-791. doi:10.1111/j.1365-2222.2012.03987.x

92. Farrant J, Brice H, Fowler S, Niven R. Fungal sensitisation in severe asthma is associated with the identification of Aspergillus fumigatus in sputum. $J$ Asthma. 2016;53(7):732-735. doi:10.3109/02770903.2016.1154073

93. Scadding AJ, Pashley CH, Monteiro W, Bhatt H, Wardlaw AJ. Cultural fungal mycobiome in non-cystic bronchiectasis. Thorax. 2018;73(Suppl 4):P8.

94. Fraczek MG, Kirwan MB, Moore CB, Morris J, Denning DW, Richardson MD. Volume dependency for culture of fungi from respiratory secretions and increased sensitivity of Aspergillus quantitative PCR. Mycoses. 2014;57(2):69-78. doi:10.1111/ myc. 12103

95. Fayemiwo S, Moore CB, Foden P, Denning DW, Richardson MD. Comparative performance of Aspergillus galactomannan ELISA and PCR in sputum from patients with ABPA and CPA. J Microbiol Methods. 2017;140:32-39. doi:10.1016/j. mimet.2017.06.016

96. Rick EM, Woolnough KF, Seear PJ, et al. The airway fungal microbiome in asthma. Clin Exp Allergy. 2020;50 (12):1325-1341. doi:10.1111/cea.13722

97. Sullivan A, Hunt EB, Ward C, et al. The presence of Aspergillus fumigatus in asthmatic airways is not clearly related to clinical disease severity. Allergy. 2020;75(5):1146-1154. doi:10.1111/ all. 14087
98. Gautier M, Normand AC, L'Ollivier C, et al. Aspergillus tubingensis: a major filamentous fungus found in the airways of patients with lung disease. Med Mycol. 2016;54(5):459-470. doi:10.1093/mmy/myv118

99. D'Hooge E, Becker P, Stubbe D, Normand AC, Piarroux R, Hendrickx M. Black aspergilli: a remaining challenge in fungal taxonomy? Med Mycol. 2019;57(6):773-780. doi:10.1093/mmy/ myy 124

100. Gago S, Overton NLD, Ben-Ghazzi N, et al. Lung colonization by Aspergillus fumigatus is controlled by ZNF77. Nat Commun. 2018;9(1):3835. doi:10.1038/s41467-018-06148-7

101. Fraczek MG, Chishimba L, Niven RM, et al. Corticosteroid treatment is associated with increased filamentous fungal burden in allergic fungal disease. J Allergy Clin Immunol. 2018;142 (2):407-414. doi:10.1016/j.jaci.2017.09.039

102. Gago S, Denning DW, Bowyer P. Pathophysiological aspects of Aspergillus colonization in disease. Med Mycol. 2019;57 (Supplement_2):S219-S27. doi:10.1093/mmy/myy076

103. Li BC, Huh SM, Prieto MD, et al. Biomarkers for the diagnosis of allergic bronchopulmonary aspergillosis in cystic fibrosis: a systematic review and meta-analysis. J Allergy Clin Immunol Pract. 2021;9. doi:10.1016/j.jaip.2020.12.064

104. O'Driscoll BR, Powell G, Chew F, et al. Comparison of skin prick tests with specific serum immunoglobulin $\mathrm{E}$ in the diagnosis of fungal sensitization in patients with severe asthma. Clin Exp Allergy. 2009;39 (11):1677-1683. doi:10.1111/j.1365-2222.2009.03339.x

105. O’Driscoll BR, Hopkinson LC, Denning DW. Mold sensitization is common amongst patients with severe asthma requiring multiple hospital admissions. BMC Pulm Med. 2005;5:4. doi:10.1186/ 1471-2466-5-4

106. Fairs A, Agbetile J, Hargadon B, et al. IgE sensitization to Aspergillus fumigatus is associated with reduced lung function in asthma. Am J Respir Crit Care Med. 2010;182(11):1362-1368. doi:10.1164/rccm.201001-0087OC

107. Menzies D, Holmes L, McCumesky G, Prys-Picard C, Niven R. Aspergillus sensitization is associated with airflow limitation and bronchiectasis in severe asthma. Allergy. 2011;66(5):679-685. doi:10.1111/j.1398-9995.2010.02542.x

108. Agarwal R, Gupta D, Aggarwal AN, Saxena AK, Chakrabarti A, Jindal SK. Clinical significance of hyperattenuating mucoid impaction in allergic bronchopulmonary aspergillosis: an analysis of 155 patients. Chest. 2007;132(4):1183-1190. doi:10.1378/ chest.07-0808

109. Kaur M, Sudan DS. Allergic Bronchopulmonary Aspergillosis (ABPA)-the High Resolution Computed Tomography (HRCT) chest imaging scenario. J Clin Diagn Res. 2014;8(6):RC05-7. doi:10.7860/JCDR/2014/8255.4423

110. Refait J, Macey J, Bui S, et al. CT evaluation of hyperattenuating mucus to diagnose allergic bronchopulmonary aspergillosis in the special condition of cystic fibrosis. J Cyst Fibros. 2019;18(4): e31-e6. doi:10.1016/j.jcf.2019.02.002

111. Horn BR, Robin ED, Theodore J, Van-kessel A. Total eosinophil counts in the management of bronchial asthma. $N$ Engl J Med. 1975;292:1152-1155. doi:10.1056/NEJM197505292922204

112. Goh KJ, Yii ACA, Lapperre TS, et al. Sensitization to Aspergillus species is associated with frequent exacerbations in severe asthma. J Asthma Allergy. 2017;10:131-140. doi:10.2147/JAA. S130459

113. Maturu VN, Agarwal R. Prevalence of Aspergillus sensitization and allergic bronchopulmonary aspergillosis in cystic fibrosis: systematic review and meta-analysis. Clin Exp Allergy. 2015;45 (12):1765-1778. doi:10.1111/cea.12595

114. Baxter CG, Moore CB, Jones AM, Webb AK, Denning DW. IgEmediated immune responses and airway detection of Aspergillus and Candida in adult cystic fibrosis. Chest. 2013;143 (5):1351-1357. doi:10.1378/chest.12-1363 
115. Pacheco A, Cuevas M, Carbelo B, et al. Eosinophilic lung disease associated with Candida albicans allergy. Eur Respir J. 1998;12 (2):502-504. doi:10.1183/09031936.98.12020502

116. Bain GA, Flower CD. Pulmonary eosinophilia. Eur J Radiol. 1996;23(1):3-8. doi:10.1016/0720-048X(96)01029-7

117. Sanchez-Alarcos JM, Martinez-Cruz R, Ortega L, Calle M, Rodriguez-Hermosa JL, Alvarez-Sala JL. ABPA mimicking bronchogenic cancer. Allergy. 2001;56(1):80-81. doi:10.1034/ j.1398-9995.2001.00840.x

118. Wardlaw AJ, Wharin S, Aung H, Shaffu S, Siddiqui S. The causes of a peripheral blood eosinophilia in a secondary care setting. Clin Exp Allerg. 2021;In Press.

119. Kirschner AN, Kuhlmann E, Kuzniar TJ. Eosinophilic pleural effusion complicating allergic bronchopulmonary aspergillosis. Respiration. 2011;82(5):478-481. doi:10.1159/000323617

120. Wark P, Wilson AW, Gibson PG. Azoles for allergic bronchopulmonary aspergillosis. Cochrane Database Syst Rev. 2000;(3): CD001108

121. Liao Q, Lam KW. Inhaled antifungal agents for the treatment and prophylaxis of pulmonary mycoses. Curr Pharm Des. 2021;26. doi:10.2174/1381612826666210101153547

122. Murray A, Cass L, Ito K, et al. PC945, a novel inhaled antifungal agent, for the treatment of respiratory fungal infections. $J$ Fungi (Basel). 2020;6(4). doi:10.3390/jof6040373.

123. Agbetile J, Bourne M, Fairs A, et al. Effectiveness of voriconazole in the treatment of Aspergillus fumigatus-associated asthma (EVITA3 study). J Allergy Clin Immunol. 2014;134(1):33-39. doi:10.1016/j.jaci.2013.09.050

124. Wark PA, Hensley MJ, Saltos N, et al. Anti-inflammatory effect of itraconazole in stable allergic bronchopulmonary aspergillosis: a randomized controlled trial. J Allergy Clin Immunol. 2003;111 (5):952-957. doi:10.1067/mai.2003.1388

125. Stevens DA, Schwartz HJ, Lee JY, et al. A randomized trial of itraconazole in allergic bronchopulmonary aspergillosis. $N \mathrm{Engl}$ JMed. 2000;342(11):756-762. doi:10.1056/NEJM200003163421102
126. Chishimba L, Niven RM, Cooley J, Denning DW. Voriconazole and posaconazole improve asthma severity in allergic bronchopulmonary aspergillosis and severe asthma with fungal sensitization. $J$ Asthma. 2012;49(4):423-433. doi:10.3109/ 02770903.2012.662568

127. Rapeport WG, Ito K, Denning DW. The role of antifungals in the management of patients with severe asthma. Clin Transl Allerg. 2020;10(1):46. doi:10.1186/s13601-020-00353-8

128. Brandt C, Roehmel J, Rickerts V, Melichar V, Niemann N, Schwarz C. Aspergillus bronchitis in patients with cystic fibrosis. Mycopathologia. 2018;183(1):61-69. doi:10.1007/ s11046-017-0190-0

129. Johnson DC. Chronic candidal bronchitis: a consecutive series. Open Respir Med J. 2012;6:145-149. doi:10.2174/ 1874306401206010145

130. Manti S, Fabio Parisi G, Papale M, et al. Allergic bronchopulmonary aspergillosis in children. Pediatr Allergy Immunol. 2020;31(Suppl 26):20-22. doi:10.1111/pai.13357

131. Sunman B, Ademhan Tural D, Ozsezen B, Emiralioglu N, Yalcin E, Ozcelik U. Current approach in the diagnosis and management of allergic bronchopulmonary aspergillosis in children with cystic fibrosis. Front Pediatr. 2020;8:582964. doi:10.3389/fped.2020.582964

132. Bush A. Kids, difficult asthma and fungus. J Fungi (Basel). 2020;6(2). doi:10.3390/jof6020055

133. Castanhinha S, Sherburn R, Walker S, et al. Pediatric severe asthma with fungal sensitization is mediated by steroid-resistant IL-33. J Allergy Clin Immunol. 2015;136(2):312-22 e7. doi:10.1016/j.jaci.2015.01.016

134. Welsh KG, Holden KA, Wardlaw AJ, et al. Fungal sensitization and positive fungal culture from sputum in children with asthma are associated with reduced lung function and acute asthma attacks respectively. Clin Exp Allergy. 2020. doi:10.1111/ cea.13799

\section{Publish your work in this journal}

The Journal of Asthma and Allergy is an international, peer-reviewed open-access journal publishing original research, reports, editorials and commentaries on the following topics: Asthma; Pulmonary physiology; Asthma related clinical health; Clinical immunology and the immunological basis of disease; Pharmacological interventions and new therapies. The manuscript management system is completely online and includes a very quick and fair peer-review system, which is all easy to use. Visit http://www.dovepress.com/testimonials.php to read real quotes from published authors. 